\title{
Global public health: a challenge for the 21st century
}

Public health is as old as the epidemic occurrence of disease, but as the nature of epidemics changed, public health constantly developed to meet the new challenges. A last major adaptation occurred when it was recognised that a number of severe chronic diseases were caused by unfavourable behaviours related to eating habits, substance abuse, and lack of physical exercise. It reached maturity with the Ottawa Charter on Health Promotion of $1986^{1}$, when the need for intersectoral action and empowerment was recognised as countermeasures to social, economic, and ecologic developments incompatible with good health. Health promotion has since primarily relied on action in local settings. Networks of healthy schools, workplaces, health facilities, and communities were developed across national borders, mainly with the aim to facilitate and support local action.

However, many of today's health problems are beyond significant influence of localised action, because they are aggravated if not caused by developments at the global level. Increasing air traffic and international transports spread infectious diseases of humans and animals across borders faster than ever. Multinational companies have introduced new products into what had been subtly balanced regional economies, weakening local production and leading to those lifestyles having previously caused chronic disease in the industrialised world. International tobacco companies bring aggressive product promotion to countries where local manufacturers had been satisfied with smaller markets. Thriving toward economic growth and shareholder value brings about stress and chronic fatigue among those having a chance to be among the winners, and frustration, social isolation, addictive behaviour, hunger and illnesses caused by poverty to the many losers. Climate change due mainly to automobile and air traffic has already led to floods and landslides in catastrophic dimensions, and threatens to bring tropical diseases in what had so far been temperate climate zones ${ }^{2}$.
Nevertheless, the threats to ecologic and physiologic balance are still developing further. Governments and standard setting bodies tend to ignore possible health hazards of innovative technology, even if these are suggested by epidemiologic research ${ }^{3}$. And even in many economically privileged parts of the world, population coverage for health care services cannot be financed, because unregulated investments in new medical technology, often by multinational companies, have reduced the resources available for primary health care.

While new public health measures will be needed at the national and local level to meet this multitude of challenges, it is much more basic in terms of sustainability to counter the threats to public health from unrestricted global economic development at their roots. International treaties such as the World Trade Agreements favouring deregulation and a free market economy across borders and cultures should be amended accordingly. International financial structures should be adapted so as to eliminate international debts and keep new debts from building $u p$. When debt relief is grant$e d$, countries should be supported in using the newly available resources to reduce poverty and develop appropriate health services for all.

How is public health organised to counter these forces? A new sub-discipline of public health is needed, which should share a political, intersectoral approach with health promotion, but act on a global rather than on a local level. As in health promotion, global health should be largely based on non governmental structures, as governments are too often slow to react to problems, and tend to overestimate the benefits of economic growth, while ignoring its less visible risks. An important step toward organised non governmental action in global public health was taken in September, 2000, in Beijing, when the World Federation of Public Health Associations (WFPHA) issued a "Call to Action", in which these problems are explicitly stated. Effective measures to 
counter uncontrolled economic globalisation were asked for; and the WFPHA was identified as a non governmental organisation well suited to assume the needed role as a catalyst. With its status as an international partner NGO of the World Health Organisation, the WFPHA can represent the values and demands of the public health movement within the United Nations system, while through its member public health associations in over sixty countries, it should be able to simultaneously stimulate discussion, political understanding and support of WHO, as well as influence policies at the national level.

The WFPHA will not be alone in trying to fill the gap in global public health action. Under the dynamic leadership of Gro Harlem Brundtland, the WHO is fast moving into this direction. It is supported by a number of international NGOs, which, however, usually lack the access to the public health communities at the national level, so that opportuni- ties for partnerships seem to offer themselves very naturally. As has been the case for all sectors of public health, the newly emerging field of global health should not be driven solely by convictions and passions, but assemble a solid scientific basis, both in terms of methodologies and knowledge. $N G O$ s and university departments wishing to engage in global health research will find that funds are available for this type of work, because some of the internationally active foundations have recognised the needs and are looking for innovative and qualified investigators ${ }^{4}$.

The WFPHA itself is developing a Plan of Action, involving research as well as action, and invites member associations and others sharing its goals to join its effort. It is hoped that this will lead in due time to a better understanding of how to take optimal advantage of globalisation in the interest of health, and to strengthening what has now tentatively been called the new discipline of global public health.

\section{References}

1 Ottawa Charter for Health Promotion. Health Promotion 1986; 1 (4): iii-iv.
2 Martens WJM, Niessen LW, Rotmans J, et al. Potential impact of global climate change on malaria risk. Environ Health Perspect 1995; 103: $458-464$.
3 Global Forum for Health Research. The $10 / 90$ report on health research 2000 . Geneva: GFHR, 2000.

Theodor Abelin 